\title{
The incidence and management of persistent cystoid macular oedema following uncomplicated cataract surgery-a Scottish Ophthalmological Surveillance Unit study
}

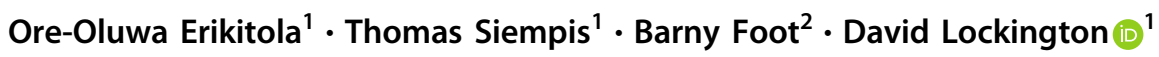

Received: 21 February 2020 / Revised: 16 April 2020 / Accepted: 16 April 2020 / Published online: 6 May 2020

(c) The Author(s), under exclusive licence to The Royal College of Ophthalmologists 2020

\begin{abstract}
Purpose Post-operative cystoid macular oedema (CMO) can cause deterioration of vision following routine cataract surgery. The incidence of persistent CMO (pCMO; defined as CMO present after 3 months) following uncomplicated surgery is uncertain. We wished to identify the incidence, management and visual outcomes of such patients.

Methods A Scottish Ophthalmological Surveillance Unit (SOSU) questionnaire was sent monthly to every ophthalmic specialist in Scotland over an 18-month period from 1st January 2018 asking them to report all new patients with pCMO confirmed on OCT scanning following uncomplicated cataract surgery. A follow-up questionnaire was sent 9 months after initial presentation.

Results Fourteen cases of pCMO were reported, giving an incidence of 2.2 cases of pCMO per 10,000 uncomplicated cataract surgeries. Mean age was 74.9 years (SD 10.2; range 44-86) with a male preponderance (72.7\%). Two patients developed pCMO in each eye. Six cases (46.2\%) had hypertension and one had diabetes. Three eyes required intracameral adjuncts (two iris hooks, one intracameral phenylephrine). Postoperative visual acuity (VA) at 3 months was $\log$ MAR 0.48 (0.2-0.8). Average mean central retinal thickness (CRT) at 3 months was 497microns (270-788). The most common initial treatment comprised topical steroids and topical NSAIDs (61.5\%). Other management strategies included systemic steroids, intravitreal steroids and oral acetazolamide. At 1-year post-op, mean VA was logMAR $0.18(0.1-0.3)$ with average mean CRT of 327microns (245-488).

Conclusions We identified a low incidence of pCMO following uncomplicated cataract surgery in Scotland $(0.02 \%)$, with inconsistent and variable management regimes. A nationally agreed treatment protocol is required.
\end{abstract}

A version of this study was an oral presentation at the Scottish Ophthalmological Club meeting in February 2020, accepted as a poster presentation at RCOphth Annual Congress, Birmingham, UK in May 2020 (postponed due to COVID-19 restrictions) and has been submitted as an oral presentation at the ESCRS annual meeting, Amsterdam, October 2020.

Supplementary information The online version of this article (https:// doi.org/10.1038/s41433-020-0908-y) contains supplementary material, which is available to authorized users.

David Lockington

davidlockington@hotmail.com

1 Tennent Institute of Ophthalmology, Gartnavel General Hospital, Glasgow, Scotland, UK

2 Royal College of Ophthalmologists, London, UK

\section{Introduction}

Cataract surgery is the most performed elective operation worldwide, with the NOD database reporting around 414,000 procedures performed in NHS England alone in 2017-2018 [1]. It is very successful, with $90.6 \%$ achieving better than driving standard vision (6/12 Snellen). Patient reported outcome measures (PROMs) consistently demonstrate significant improvements in patients' experiences and quality of life [2]. However, poorer visual outcomes occur infrequently due to complications during surgery (often associated with posterior capsule rupture and vitreous loss) or in the postoperative period (such as endophthalmitis, retinal detachment and cystoid macular oedema (CMO)) [3, 4].

CMO can also occur in the setting of uncomplicated cataract surgery, with published incidence rates ranging from $1.2-3.4 \%$ [5-10]. Known as Irvine-Gass syndrome for the past 60 years, patients commonly experience good 
vision in the immediate post-operative period, followed by a painless central visual deterioration a few weeks later [7]. CMO is believed to result from a post-operative inflammatory response [11]. The incidence of postoperative CMO peaks at 4-6 weeks, but most cases are self-limiting, though visual impairment can persist, with duration of CMO reported to range between 72 and 249 days [12]. Aside from the personal impact of visual impairment, the overall Medicare cost of modern cataract surgery complicated by CMO has been reported to be $47 \%$ higher than for those without [13]. It is generally accepted that the incidence of CMO has reduced significantly due to refinement in cataract surgical techniques, including the switch from extracapsular cataract surgery to phacoemulsification [14-16]. However, this incidence is variable, and further influenced by type (acute/chronic/ persistent) and method of detection (clinical, angiographic, optical) [11]. For example, the incidence of angiographic CMO has been previously estimated to be as high as $30 \%$ in uncomplicated cataract surgery cases [11]. In addition, the widespread use of optical coherence tomography (OCT) has increased the ability to detect both clinical and sub-clinical CMO, which could further influence quoted incidence rates [10, 17].

There is no consistent definition for post-operative CMO in either the historic or recently published literature [9]. Traditionally, most clinicians would define clinically significant $\mathrm{CMO}$ as a symptomatic reduction in vision equivalent to $20 / 40(6 / 12$ Snellen) or worse following routine cataract surgery, as observed in $<1 \%$ of patients after cataract surgery in the absence of any treatment [18]. There is no consistency as to the definition of prolonged $\mathrm{CMO}$, although persistent cystoid macular oedema (pCMO) or late onset macular oedema has been defined as the presence of macular oedema over 3 months since the original cataract surgery [12].

The ESCRS PREvention of Macular EDema after cataract surgery (PREMED) study was the first international multi-centre randomised controlled clinical trial specifically designed to answer questions relating to the prevention of CMO after cataract surgery in diabetic and non-diabetic patients $[9,10]$. They also highlighted the difficulties in defining CMO and the issues with diagnosis. This landmark study focused on the prevention of CMO through preoperative topical medications, with outcome measures at both 6- and 12-weeks post cataract surgery. It did not investigate the post-operative management of CMO or continue to identify the incidence of pCMO at 3 months. It should also be noted that there are no published national college or cataract society guidelines as to the best practice management of post-operative CMO or pCMO.

The primary aim of this Scottish Ophthalmological Surveillance Unit (SOSU) study was to determine the incidence of pseudophakic pCMO documented on OCT over 3 months from the date of uncomplicated cataract surgery in Scotland.

\section{Methods}

All new cases of pCMO identified by consultant ophthalmologists in Scotland were collected prospectively between 1 January 2018 and 31 June 2019 via the Scottish Ophthalmological Surveillance Unit (SOSU) scheme. SOSU is administered alongside the British Ophthalmological Surveillance Unit (BOSU) via the Royal College of Ophthalmologists in London (RCOphth) and has the primary aim to assist with the epidemiological study of rare ocular disorders. BOSU was originally established in 1997. Participation in the scheme is in good faith and is not mandatory. Prior to the initiation of a study, the BOSU/SOSU committee distribute the details of the upcoming conditions under surveillance, including the agreed case definition. Suitable conditions for SOSU studies are those with a predicted annual incidence of less than 30 per million $(<150$ cases per annum in Scotland). These studies then rely on ophthalmologists to voluntarily keep a record of the relevant cases they encounter during the study period, and then respond to two separate questionnaires with answers obtained from the relevant medical casenotes. The patients are anonymous to the SOSU/BOSU investigators, and there is no system to further investigate the patients' data aside from the data returned on the questionnaires.

Each month during the study period, every ophthalmologist in Scotland was posted a SOSU reporting card asking if they had managed any new cases of pCMO in their practice. When a case was reported, a questionnaire was sent to the notifying ophthalmologist asking for predetermined demographic and relevant pre-, intra- and post-operative clinical data. [See online Supplementary Figure for initial questionnaire]

If the initial questionnaire was not returned within 6 weeks, a reminder letter and a follow up email was sent to increase the response rate. Reporting ophthalmologists were also sent a follow-up questionnaire 9 months later to obtain 1 -year post-operative outcomes.

Ethics advice was sought from the West of Scotland Research and Ethics Service and approval was granted through the Public Benefit and Privacy Panel for Health and Social Care in Scotland (Reference number: 1718-0096).

\section{Outcome measures}

The primary outcome of the study was to determine the incidence of persistent cystoid macular oedema (pCMO), documented on optical coherence topography (OCT), 
$\geq 3$ months from the date of uncomplicated cataract surgery in Scotland. CMO was defined as an increase in CRT of $10 \%$ or more over baseline, with cystic changes on OCT as identified by the reporting ophthalmologist. Secondary outcomes were to ascertain the associated clinical and demographic features contributing to pCMO, to describe what management options are employed in the treatment of pCMO and to establish visual outcomes for patients with pCMO at 1-year post surgery.

\section{Results}

A total of 22 responses were received over the 18-month period, with a true response rate of $64 \%$. Eight responses were found to have been sent in error by reporting ophthalmologists, all of whom had ticked the wrong box on the reporting card.

\section{Population and Incidence}

The incidence was calculated as the number of reported cases as a proportion of the total population over the 18month study. Scotland has been recognised in previous studies as a well-defined region with stable population demographics [19]. According to the information services division of NHS National Services Scotland, the number of cataract surgeries performed in Scotland over the last three financial years (1st April to 31st March) has been 42,593 in 2014/15, 42,573 in 2015/16 and 41,336 in 2016/17 [20].

Fourteen cases of pCMO were reported, giving an incidence of 2.2 cases of pCMO per 10,000 of uncomplicated cataract surgeries per annum (0.02\%). Complete data was available for 13 cases (11 patients). Mean age was 74.9 years \pm SD 10.2 (range 44-86) with males accounting for the majority of cases (72.7\%). $92.3 \%$ of the patients were Caucasian. Two patients developed pCMO in both eyes following uncomplicated cataract surgery.

Most patients presented directly back to hospital eye services (61.5\%). In Scotland, optometrists are responsible for primary eye care services, and $31.8 \%$ of patients initially presented to them. The most common presenting complaint was reduction in vision (10 patients; $76.9 \%)$. The other three patients with pCMO were asymptomatic on presentation but were picked up on refraction by the optometrist $(23 \%)$. Mean time of presentation to hospital eye services from date of original uncomplicated surgery was 85.4 days (range 10-157 days). (See Fig. 1)

Six cases $(46.2 \%)$ had pre-existing hypertension and two cases $(15.4 \%)$ had pre-existing glaucoma. Only one patient had known diabetes mellitus. None of the patients had a previous history of uveitis or were using topical prostaglandin analogues.

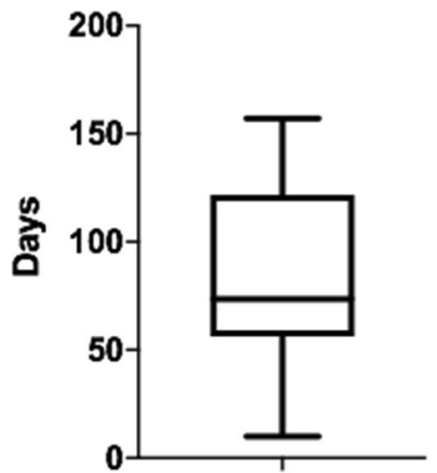

Fig. 1 Graph illustrating time to diagnosis of persistent CMO, showing delay in presentation by patients following routine cataract surgery.

Six eyes (46.2\%) had their surgery performed under topical anaesthesia. Other methods included sub-Tenon's injection of local anaesthetic (38.5\%) and general anaesthesia $(7.7 \%)$. Three eyes $(23.1 \%)$ required intracameral adjuncts intra-operatively (two with iris hooks (15.4\%), one with intracameral phenylephrine $(7.7 \%)$ ). In the two patients who developed pCMO in both eyes, both patients had surgery performed under sub-Tenon's anaesthesia and at least one eye had required iris hooks intra-operatively.

Only one patient received a subconjunctival steroid injection at the end of the surgery.

\section{Management and outcomes}

Table 1 details the first line treatments employed at initial presentation and subsequent management strategies.

Average mean central retinal thickness (CRT) at 3 months post cataract surgery was 497 microns (range 270-788 $\mu \mathrm{m}$ ) for 10 eyes. At 1-year post op review, average mean CRT was 327 microns (range 245-488 $\mu \mathrm{m}$ ). Five eyes still had pCMO on OCT scanning. Of these five cases, three had evidence of epi-retinal membrane. The difference between CRT at presentation (3 months following cataract surgery) and at 1-year post op for 10 patients was not statistically significant $(p=0.27)$ (Two-way ANOVA). (See Fig. 2) The mean visual acuity (VA) at 3 months postsurgery for all 14 patients was LogMAR 0.48 (range 0.2-0.8). At 1-year post op, average VA was LogMAR 0.18 (range 0.1-0.3). A clinically significant difference in BCVA as defined as an improvement of $>2$ Snellen lines at 1-year post op was identified in five patients, but this wasn't found to be statistically significant $(p=0.19) \quad$ (Two-way ANOVA). (See Fig. 3)

\section{Discussion}

Previous studies have reported an incidence of $1.2-2.0 \%$ in patients with no risk factors for developing clinically 
Table 1 Table detailing first line treatments employed at initial presentation of persistent $\mathrm{CMO}$ at 3 months, and subsequent management strategies within one year following cataract surgery.

\begin{tabular}{lll}
\hline Treatment & 3 months post op & 1 year post op \\
\hline $\begin{array}{l}\text { Combination of topical steroids and topical non-steroidal } \\
\text { anti-inflammatory drugs (NSAIDs) }\end{array}$ & 8 cases $(61.5 \%)$ & 4 cases $(30.8 \%)$ \\
Topical NSAIDs only & 2 cases $(15.4 \%)$ & 1 case $(7.7 \%)$ \\
Topical steroid only & 1 case $(7.7 \%)$ & 1 case $(7.7 \%)$ \\
Topical steroids, topical NSAIDs and oral Acetazolamide & 1 case $(7.7 \%)$ & 1 case $(7.7 \%)$ \\
Topical steroid, topical NSAIDs and oral Prednisolone & 1 case $(7.7 \%)$ & - \\
Observation & - & 3 cases $(23.1 \%)$ \\
Intravitreal steroid implant (Ozurdex) & - & 1 case $(7.7 \%)$ \\
Sub-Tenon's triamcinolone injection & - & 1 case $(7.7 \%)$ \\
Vitreoretinal referral & - & 1 case $(7.7 \%)$ \\
\hline
\end{tabular}

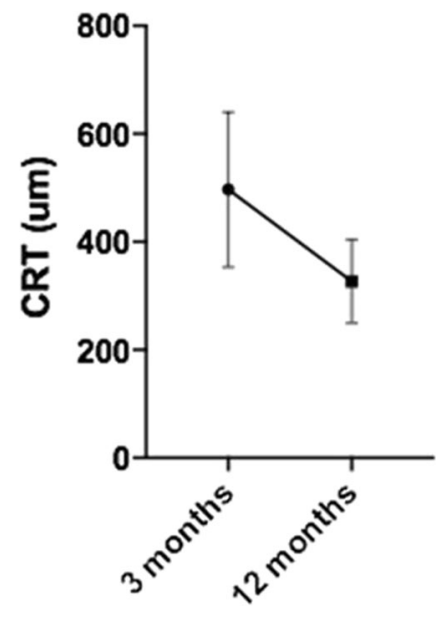

Fig. 2 Graph comparing central retinal thickness (CRT) over time, showing reduction in OCT measured CRT in patients with persistent $\mathrm{CMO}$ at 3 months and 1 year following cataract surgery.

significant CMO after cataract surgery [4, 7]. However, there are no robust definition or incidence figures regarding persistent CMO. (12) This prospective study from an established national surveillance scheme investigating all cases of persistent pseudophakic cystoid macular oedema at 3 months following uncomplicated cataract surgery in Scotland identified 14 patients, which equates to an incidence rate of 2.2 in 10,000 cases $(0.02 \%)$.

Similar to published studies of pseudophakic CMO, our cases of pCMO tended to occur in older (average age 74.9 years) and male $(72.7 \%)$ patients $[4,12,13]$. It is unclear from the literature why the male gender carries a higher risk, although research identifying gender differences in ocular blood flow was consistent with the Gutenberg Health Study which found men to be 1.7 times more likely to develop retinal vein occlusions than females [21, 22].

Nearly half $(46.2 \%)$ of our cohort had systemic hypertension. Interestingly, no large study has evaluated the link between hypertension and development of pCMO. It is well known that systemic hypertension causes arteriosclerosis and leakage from the retinal blood vessels and predisposes to

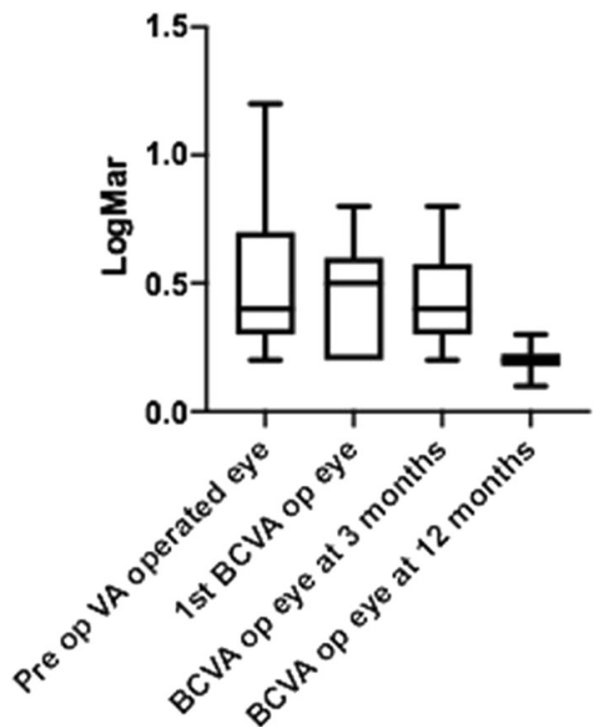

Fig. 3 Graph comparing changes in best corrected visual acuity (BCVA) in patients with persistent CMO over the 12 months following cataract surgery.

retinal vein occlusions [23]. To illustrate this association, Kida et al. reported a case series of three patients with systemic hypertension without any other risk factors who developed macular oedema associated with retinal vein occlusions. All three cases showed improvement in the macular oedema after treatment of the systemic hypertension alone [24].

Use of topical prostaglandin analogues has been implicated in increasing the risk of CMO [8, 12]. A small case series of randomised patients to topical prostaglandin analogues (bimatoprost, latanoprost, and travoprost) noted 6 (12.5\%) patients subsequently developed angiographic CMO, although only one had an associated loss of visual acuity [25]. All these cases of angiographic pseudophakic CMO subsequently resolved on cessation of prostaglandin analogues and commencement of topical diclofenac [25]. However, other groups have reported no significant increase in clinical CMO with topical prostaglandin analogues [3, 4]. Interestingly, our two patients with glaucoma were being 
treated with topical beta-blockers rather than prostaglandin analogues. Wendel et al. showed that apart from prostaglandin analogues, topical beta-blockers are also associated with the development of pseudophakic CMO. Through analysis of 508 cases and 5080 controls, they found the incidence of pseudophakic CMO to be statistically significantly associated with the current postoperative use of both topical PGAs [relative risk (RR), 1.86; 95\% confidence interval (CI), 1.04-3.32] and topical betablockers (RR, 2.64; 95\% CI, 1.08-6.49) [8].

Inflammation remains the most commonly accepted contributor to the development of pseudophakic CMO [11, 16, 26-29]. Surgical manipulation of the iris can result in an upregulation of inflammatory mediators, leading to breakdown of the blood-retinal-barrier and increased vascular permeability [11]. This inflammation is associated with abnormal accumulation of intracellular and extracellular fluid that results in the classic cystic changes in the outer plexiform layer and inner nuclear layer of the macula with associated retinal thickening. In our cohort, two patients had intra-operative adjuncts in the form of iris hooks which could have contributed to an enhanced inflammatory response in the post-operative period.

Studies have found a fourfold increase in the incidence of pseudophakic CMO in patients with diabetes, with risk of development linked to the degree of ETDRS-graded severity of diabetic retinopathy [4]. These findings are consistent with a deficient blood-retinal barrier function in those patients with more advanced diabetic retinopathy vascular changes. Recent retrospective studies have documented incidence rates of postoperative CMO in diabetic patients to be between $0.1 \%$ and $2.35 \%[12,30]$. In our study only one patient had diabetes (without active diabetic retinopathy or maculopathy before cataract surgery). This patient did not receive any prophylactic topical NSAIDs. A report by the American Academy of Ophthalmology in 2015 advised that peri-operative topical NSAIDs are effective in reducing the occurrence of pseudophakic CMO, may increase the speed of visual recovery postoperatively in diabetic patients, but do not alter long-term visual outcomes beyond 3 months [5].

\section{Lack of evidence for treatment options}

Currently, there are no UK nationally agreed guidelines for the treatment of acute or chronic post-operative CMO from either the Royal College of Ophthalmologists or national cataract societies (such as UKISCRS (United Kingdom and Ireland Society of Cataract and Refractive Surgeons; see www.ukiscrs.org.uk)). The published literature suggested a variety of topical and systemic treatment modalities. These include use of topical NSAIDs, topical steroids, combination of topical NSAIDs and steroids, periocular steroid injections, systemic steroids, systemic carbonic anhydrase inhibitors, intravitreal injections of anti-Vascular Endothelial Growth Factor agents (anti-VEGF), intravitreal steroid injections, intravitreal steroid implants, and surgery in the form of pars plana vitrectomy [6, 7, 9, 14, 26, 31-37]. A recent review of CMO by McGhee's group in Auckland concluded that "there are limited evidence-based clinical studies available regarding successful treatment strategies, and there is substantial inconsistency between studies in terms of defining successful outcome measures, with some focusing on improvements as measured by OCT or fluorescein angiography vs visual acuity outcomes" [29]. They noted that visual improvement doesn't always correlate with angiographic improvement, and spontaneous resolution of CMO can be a major confounder in any attempted analysis of true treatment effects [29, 38].

The most common initial strategy for treatment of postoperative $\mathrm{CMO}$ in our study involved combination of topical steroids and topical NSAIDs in eight cases (61.5\%), although another patient had this regime supplemented with oral acetazolamide and another had supplementation with oral steroids. In total, 10 cases $(76.9 \%)$ were initially treated with a strategy involving combined topical NSAIDs and steroids (with or without additional systemic medications) [38].

As previously noted, the association of pseudophakic CMO with inflammation led the ESCRS PREMED study group to compare the efficacy of a topical NSAID, topical corticosteroid, and a combination of both drugs to prevent the occurrence of CMO after cataract surgery in nondiabetic patients [9]. In this large RCT study investigating prevention (not treatment) of 914 patients, they discovered that patients treated with a combination of topical NSAID (bromfenac 0.09\%) and topical steroid (dexamethasone $0.1 \%$ ) had a lower risk for developing clinically significant macular oedema after cataract surgery than patients treated with a single drug. However, it should be noted that this study ended at 12 weeks and did not continue onto evaluate any patients with pCMO. While our study patients (looking at treatment) are different to the PREMED group (looking at prevention), $50 \%$ of our eight cases on combination topical NSAIDs and steroids treatment had resolution of CMO at 1year post-op follow up. Of the other four remaining cases with pCMO, three had evidence of epi-retinal membrane causing cystic spaces on OCT scans at 1-year post-op.

It is tempting to try to extrapolate the PREMED study results to make recommendations as to treatment of patients with CMO, but that was not their intention and so caution should be exercised. Equally, there has not been a detailed cost analysis evaluating the financial implications of a prevention versus treatment strategy for post-operative CMO. Such a study may be difficult to perform due to the variability in management strategies, but would be 
useful to guide treatment, as there is a significant additional cost to frequent return clinical appointments following cataract surgery (47\% higher costs as per Schiemer) [13].

Periocular and orbital floor corticosteroid injection were traditionally used to treat $\mathrm{pCMO}$ unresponsive to topical treatment, yet we only identified one patient who underwent this management strategy. A small prospective study by Thach et al. of 48 eyes with clinically significant persistent CMO treated with sub-Tenon's triamcinolone produced an improvement in BCVA and central retinal thickness. The posterior sub-Tenon's group had a visual improvement from $20 / 92$ pretreatment to $20 / 50$ post-treatment $(P=0.0001)$ with a median follow-up of 12 months [31]. Similarly, a small retrospective study of six eyes with unresponsive CMO reported visual improvement with significant reduction of retinal thickness following $40 \mathrm{mg}$ of orbital floor triamcinolone injection at mean follow up of 11 months [39].

The evidence supporting the use of systemic acetazolamide in the treatment of CMO is limited. Curkovic et al. in a small, randomised controlled trial of 14 eyes, studied the efficacy of oral acetazolamide $250 \mathrm{mg}$ three times daily in addition to topical dexamethasone and flurbiprofen. They reported complete resolution of PCMO in $86 \%$ of eyes receiving acetazolamide (plus the topical NSAID-steroid combination) vs $29 \%$ in the control group who received topical dexamethasone and flurbiprofen alone [40]. This treatment regime was not seen to be effective in the single patient who also received oral acetazolamide in our study.

Slow release intravitreal dexamethasone implants show promise in the treatment of chronic pseudophakic CMO. Mayer et al. reported an improvement in visual acuity, foveal thickness and retinal sensitivity in a prospective study of 23 eyes treated with a dexamethasone implant (Ozurdex) with 12 month follow-up [35]. Recurrence was noted in nine eyes with a peak after 3 months and these eyes required a second implant. Bellocq et al. in a multi-centre national case series of 100 eyes receiving a dexamethasone $0.7 \mathrm{mg}$ implant for postsurgical macular oedema, noted that $37 \%$ of patients required only one dexamethasone implant during the first year and experienced no recurrence of the macular oedema in a follow-up period of greater than 1 year. The significant improvement in visual acuity at 6 months was maintained at 12 months following treatment [6].

It has been our observation that use of a combined subconjunctival injection of antibiotic and corticosteroid at the end of the cataract operation used to be common clinical practice. We wonder if the advent of intracameral antibiotic use may have also resulted in the inadvertent omission of the peri-operative steroid bolus. The evidence to support the efficacy of peri-operative subconjunctival steroid is limited, but it is notable that this step is now often omitted in modern cataract surgery. Mirroring this change in clinical practice, we identified only one case who had received subconjunctival steroid injection in our cohort (i.e. $92.3 \%$ of our cases of pCMO did not receive subconjunctival perioperative steroid at the end of surgery).

\section{Long-term visual outcomes}

CMO can resolve, but still result in permanent visual loss [4, 34]. Hunter's group reported $26.8 \%$ of eyes with pseudophakic CMO didn't recover 6/6 vision [41]. In our cohort, 11 cases didn't achieve this visual outcome. At 1year post-op, three of our patients had the presence of an ERM. It is possible that these patients had a pre-existing ERM which was not identified because it is not common NHS practice in Scotland to perform macula OCT scanning in all pre-operative cases.

\section{Limitations of this study}

This SOSU study has many limitations that are intrinsically related to the study design and methodology. Such BOSU/ SOSU surveillance studies are widely recognised to result in lower incidence rates than in real-life due to the inherent potential for under-reporting by observers [19]. Furthermore, because routine post-operative cataract reviews have been devolved to community optometry in Scotland, the identification rate may be lower from optometric practices without OCT. The SOSU methodology meant our study could only identify patients who had been referred back into the hospital system and were under the care of an ophthalmologist. We also have no knowledge of optometric independent prescribing behaviours, which could also have suppressed the incidence numbers. Our study had a followup of 18 months postoperatively, yet only identified 14 cases. It is difficult to develop management recommendations from such a small number. The patients originated from different Scottish Ophthalmology departments which use different OCT devices, which could influence the reporting of macular thickness, as some machines measure retinal thickness from the internal limiting membrane to the retinal pigment epithelium (RPE), while others use Bruchs membrane as the outer retinal boundary. It must be recognised that OCT interpretation on different machines is not standardised across the Scottish ophthalmic departments. Equally, it is not possible to standardise the follow up and review arrangements in each hospital for these patients, so we had to use the closest appointment information for the 3 month and 12 month post-operative data. In addition, bias due to failure to report can negatively impact any survey regarding the incidence of surgical complications, as has been previously reported in the literature looking at the validity of reporting capsule rupture data events [42]. However, while these issues may influence the true 
incidence in our study, we believe this SOSU study does provide accurate information as to the real-world current management of patients with persistent CMO post routine cataract surgery in Scotland.

The current available published literature does not provide clear evidence with regard to the long-term efficacy of topical NSAIDs and/or corticosteroids for CMO on BCVA more than 3 months after routine cataract surgery, which is beyond the 12 weeks follow up timeframe of the PREMED studies $[9,10]$. This gap in the literature could provide further incentive for a larger UK wide (possibly BOSU) prospective study to identify more patients with longer follow up periods and more standardised review arrangements to try and identify best practice in this patient group.

\section{Conclusion}

We have identified a low incidence of pCMO following uncomplicated cataract surgery in Scotland $(0.02 \%)$. This is probably the lowest estimate, as underreporting is a common issue with SOSU/BOSU surveillance surveys and routine post-operative cataract reviews have been devolved to community optometrists in Scotland. Reported clinical management regimes for patients with pCMO were inconsistent and variable. We would suggest a wider prospective UK study may identify more cases. We believe a nationally agreed Royal College or national cataract society (e.g. UKISCRS) protocol for the treatment of post-operative pCMO following routine cataract surgery is now required.

\section{Summary}

\section{What was known before}

- There is great variation and inconsistency as to the definition of cystoid macular oedema (CMO) following routine cataract surgery

- There are no studies identifying the incidence of persistent $\mathrm{CMO}$ at 3 months (pCMO) following routine cataract surgery.

- There are no formal national cataract society or Royal College recommendations for best management of such cases

\section{What this study adds}

- This prospective SOSU study has identified a low incidence of pCMO following uncomplicated cataract surgery in Scotland $(0.02 \%)$.

- Reported clinical management regimes for patients with pCMO are inconsistent and variable.
- We would suggest a wider UK prospective study may identify more cases and believe a nationally agreed Royal College or national cataract society (e.g. UKISCRS) protocol for the treatment of post-operative pCMO following routine cataract surgery is now required.

Acknowledgements We thank the SOSU committee for their guidance and support in this project. We thank the Ross Foundation for providing funding through their bursary. We wish to acknowledge the following ophthalmologists who contributed patients to the study: Dr Boyle, Dr Cobb, Dr Drummond, Dr Fairlie, Dr Gillan, Dr Gilmour, Dr MacLeod, Dr MacRae, Dr Pyott, Dr Rotchford and Dr Scott.

\section{Compliance with ethical standards}

Conflict of interest The authors declare that they have no conflict of interest.

\section{Consent for publication Granted.}

Publisher's note Springer Nature remains neutral with regard to jurisdictional claims in published maps and institutional affiliations.

\section{References}

1. Royal College of Ophthalmologists (UK). National ophthalmology database audit. 2019. https://nodaudit.org.uk/u/docs/20/ urxqilwxmv/NOD\%20Audit\%20Annual\%20Report\%202019.pdf, Accessed 4 Feb 2020.

2. Busbee BG, Brown MM, Brown GC, Sharma S. Incremental costeffectiveness of initial cataract surgery. Ophthalmology. 2002;109:606-12.

3. Law SK, Kim E, Yu F, Caprioli J. Clinical cystoid macular edema after cataract surgery in glaucoma patients. J Glaucoma. 2010;19:100-4.

4. Chu CJ, Johnston RL, Buscombe C, Sallam AB, Mohamed Q, Yang YC. Risk factors and incidence of macular edema after cataract surgery: a database study of 81984 eyes. Ophthalmology. 2016;123:316-23.

5. Kim SJ, Schoenberger SD, Thorne JE, Ehlers JP, Yeh S, Bakri SJ. Topical nonsteroidal anti-inflammatory drugs and cataract surgery: a report by the American Academy of Ophthalmology. Ophthalmology. 2015;122:2159-68.

6. Bellocq D, Pierre-Kahn V, Matonti F, Burillon C, Voirin N, Dot $\mathrm{C}$, et al. Effectiveness and safety of dexamethasone implants for postsurgical macular oedema including Irvine-Gass syndrome: the EPISODIC-2 study. Br J Ophthalmol. 2017;101:333-41.

7. McCafferty S, Harris A, Kew C, Kassm T, Lane L, Levine J, et al. Pseudophakic cystoid macular edema prevention and risk factors; prospective study with adjunctive once daily topical nepafenac 0.3\% versus placebo. BMC Ophthalmol. 2017;17:16.

8. Wendel C, Zakrzewski H, Carleton B, Etminan M, Mikelberg FS. Association of postoperative topical prostaglandin analog or betablocker use and incidence of pseudophakic cystoid macular edema. J Glaucoma. 2018;27:402-6.

9. Wielders LHP, Schouten J, Winkens B, van den Biggelaar F, Veldhuizen CA, Findl O, et al. European multicenter trial of the prevention of cystoid macular edema after cataract surgery in nondiabetics: ESCRS PREMED study report 1. J Cataract Refract Surg. 2018;44:429-39. 
10. Wielders LHP, Schouten J, Winkens B, van den Biggelaar F, Veldhuizen CA, Murta JCN, et al. Randomized controlled European multicenter trial on the prevention of cystoid macular edema after cataract surgery in diabetics: ESCRS PREMED Study Report 2. J Cataract Refract Surg. 2018;44:836-47.

11. Flach AJ. The incidence, pathogenesis and treatment of cystoid macular edema following cataract surgery. Trans Am Ophthalmol Soc. 1998;96:557-634.

12. Henderson BA, Kim JY, Ament CS, Ferrufino-Ponce ZK, Grabowska A, Cremers SL. Clinical pseudophakic cystoid macular edema. Risk factors for development and duration after treatment. J Cataract Refract Surg. 2007;33:1550-8.

13. Schmier JK, Halpern MT, Covert DW, Matthews GP. Evaluation of costs for cystoid macular edema among patients after cataract surgery. Retina. 2007;27:621-8.

14. Karacorlu M, Ozdemir H, Karacorlu S. Intravitreal triamcinolone acetonide for the treatment of chronic pseudophakic cystoid macular oedema. Acta Ophthalmol Scand. 2003;81:648-52.

15. Yonekawa Y, Kim IK. Pseudophakic cystoid macular edema. Curr Opin Ophthalmol. 2012;23:26-32.

16. Grzybowski A, Sikorski BL, Ascaso FJ, Huerva V. Pseudophakic cystoid macular edema: update 2016. Clin Inter Aging. 2016;11:1221-9.

17. Puliafito CA, Hee MR, Lin CP, Reichel E, Schuman JS, Duker JS, et al. Imaging of macular diseases with optical coherence tomography. Ophthalmology. 1995;102:217-29.

18. Guo S, Patel S, Baumrind B, Johnson K, Levinsohn D, Marcus E, et al. Management of pseudophakic cystoid macular edema. Surv Ophthalmol. 2015;60:123-37.

19. Foot B, Stanford M, Rahi J, Thompson J. The British Ophthalmological Surveillance Unit: an evaluation of the first 3 years. Eye (Lond). 2003;17:9-15.

20. Surgical Procedures. Information Services Division. NHS National Services Scotland. 2019. Accessed April 2020 at https://www.isdscotland.org/products-and-services/scottish-atlasof-variation/view-the-atlas/surgical-procedures.asp.

21. Rath EZ, Frank RN, Shin DH, Kim C. Risk factors for retinal vein occlusions. A case-control study. Ophthalmology. 1992;99:509-14.

22. Ponto KA, Elbaz H, Peto T, Laubert-Reh D, Binder H, Wild PS, et al. Prevalence and risk factors of retinal vein occlusion: the Gutenberg Health Study. J Thromb Haemost. 2015;13:1254-63.

23. Martinez F, Furio E, Fabia MJ, Perez AV, Gonzalez-Albert V, Rojo-Martinez G, et al. Risk factors associated with retinal vein occlusion. Int J Clin Pr. 2014;68:871-81.

24. Kida T, Morishita S, Kakurai K, Suzuki H, Oku H, Ikeda T. Treatment of systemic hypertension is important for improvement of macular edema associated with retinal vein occlusion. Clin Ophthalmol. 2014;8:955-8.

25. Arcieri ES, Santana A, Rocha FN, Guapo GL, Costa VP. Bloodaqueous barrier changes after the use of prostaglandin analogues in patients with pseudophakia and aphakia: a 6-month randomized trial. Arch Ophthalmol. 2005;123:186-92.

26. Flach AJ, Dolan BJ, Irvine AR. Effectiveness of ketorolac tromethamine $0.5 \%$ ophthalmic solution for chronic aphakic and pseudophakic cystoid macular edema. Am J Ophthalmol. 1987; 103:479-86.
27. Loewenstein A, Zur D. Postsurgical cystoid macular edema. Dev Ophthalmol. 2010;47:148-59.

28. Lobo C. Pseudophakic cystoid macular edema. Ophthalmologica. 2012;227:61-7.

29. Han JV, Patel DV, Squirrell D, McGhee CN. Cystoid macular oedema following cataract surgery: A review. Clin Exp Ophthalmol. 2019;47:346-56.

30. Packer M, Lowe J, Fine H. Incidence of acute postoperative cystoid macular edema in clinical practice. J Cataract Refract Surg. 2012;38:2108-11.

31. Thach AB, Dugel PU, Flindall RJ, Sipperley JO, Sneed SR. A comparison of retrobulbar versus sub-Tenon's corticosteroid therapy for cystoid macular edema refractory to topical medications. Ophthalmology. 1997;104:2003-8.

32. Sivaprasad S, Bunce C, Wormald R. Non-steroidal antiinflammatory agents for cystoid macular oedema following cataract surgery: a systematic review. Br J Ophthalmol. 2005;89: $1420-2$.

33. Arevalo JF, Maia M, Garcia-Amaris RA, Roca JA, Sanchez JG, Berrocal $\mathrm{MH}$, et al. Intravitreal bevacizumab for refractory pseudophakic cystoid macular edema: the Pan-American Collaborative Retina Study Group results. Ophthalmology. 2009;116:1481-7, 7.e1.

34. Kessel L, Tendal B, Jorgensen KJ, Erngaard D, Flesner P, Andresen JL, et al. Post-cataract prevention of inflammation and macular edema by steroid and nonsteroidal anti-inflammatory eye drops: a systematic review. Ophthalmology. 2014;121:1915-24.

35. Mayer WJ, Kurz S, Wolf A, Kook D, Kreutzer T, Kampik A, et al. Dexamethasone implant as an effective treatment option for macular edema due to Irvine-Gass syndrome. J Cataract Refract Surg. 2015;41:1954-61.

36. Juthani VV, Clearfield E, Chuck RS. Non-steroidal anti-inflammatory drugs versus corticosteroids for controlling inflammation after uncomplicated cataract surgery. Cochrane Database Syst Rev. 2017;7:Cd010516.

37. Yuksel B, Uzunel UD, Kerci SG, Sagban L, Kusbeci T, Orsel T. Comparison of subtenon triamcinolone acetonide injection with topical nepafenac for the treatment of pseudophakic cystoid macular edema. Ocul Immunol Inflamm. 2017;25:513-9.

38. Jacobson DR, Dellaporta A. Natural history of cystoid macular edema after cataract extraction. Am J Ophthalmol. 1974;77:445-7.

39. Suleman H, Mathew M, Lakshmanan A, Abedin A, Orr G. Orbital floor triamcinolone acetonide in the treatment of pseudophakic cystoid macular oedema. Acta Ophthalmol. 2008;86. https://doi. org/10.1111/j.1755-3768.2008.6417.x.

40. Curkovic T, Vukojevic N, Bucan K. Treatment of pseudophakic cystoid macular oedema. Coll Antropol. 2005;29:103-5.

41. Hunter AA, Modjtahedi SP, Long K, Zawadzki R, Chin EK, Caspar JJ, et al. Improving visual outcomes by preserving outer retina morphology in eyes with resolved pseudophakic cystoid macular edema. J Cataract Refract Surg. 2014;40:626-31.

42. Lundström M, Behndig A, Kugelberg M, Montan P, Stenevi U, Thorburn W. Decreasing rate of capsule complications in cataract surgery: eight-year study of incidence, risk factors, and data validity by the Swedish National Cataract Register. J Cataract Refract Surg. 2011;37:1762-7. 